\title{
Dynamic glass transition in two dimensions
}

\author{
M. Bayer, ${ }^{1}$ J. M. Brader, ${ }^{1}$ F. Ebert, ${ }^{1}$ M. Fuchs, ${ }^{1}$ E. Lange, ${ }^{1}$ G. Maret, ${ }^{1}$ R. Schilling, ${ }^{2}, *$ M. Sperl, ${ }^{3}$ and J. P. Wittmer ${ }^{4}$ \\ ${ }^{1}$ Fachbereich Physik, Universität Konstanz, 78457 Konstanz, Germany \\ ${ }^{2}$ Institut für Physik, Johannes Gutenberg-Universität Mainz, Staudinger Weg 7, D-55099 Mainz, Germany \\ ${ }^{3}$ Institut für Materialphysik im Weltraum, Deutsches Zentrum für Luft-und Raumfahrt, 51170 Köln, Germany \\ ${ }^{4}$ Institut Charles Sadron, 6 rue Boussingault, 67083 Strasbourg, France
}

(Received 7 March 2007; published 20 July 2007)

\begin{abstract}
The question of the existence of a structural glass transition in two dimensions is studied using mode coupling theory (MCT). We determine the explicit $d$ dependence of the memory functional of mode coupling for one-component systems. Applied to two dimensions we solve the MCT equations numerically for monodisperse hard disks. A dynamic glass transition is found at a critical packing fraction $\varphi_{c}^{d=2} \cong 0.697$ which is above $\varphi_{c}^{d=3} \cong 0.516$ by about $35 \%$. $\varphi_{c}^{d}$ scales approximately with $\varphi_{\text {rcp }}^{d}$, the value for random close packing, at least for $d=2,3$. Quantities characterizing the local, cooperative "cage motion" do not differ much for $d=2$ and $d=3$, and we, e.g., find the Lindemann criterion for the localization length at the glass transition. The final relaxation obeys the superposition principle, collapsing remarkably well onto a Kohlrausch law. The $d=2 \mathrm{MCT}$ results are in qualitative agreement with existing results from Monte Carlo and molecular dynamics simulations. The mean-squared displacements measured experimentally for a quasi-two-dimensional binary system of dipolar hard spheres can be described satisfactorily by MCT for monodisperse hard disks over four decades in time provided the experimental control parameter $\Gamma$ (which measures the strength of dipolar interactions) and the packing fraction $\varphi$ are properly related to each other.
\end{abstract}

DOI: 10.1103/PhysRevE.76.011508

PACS number(s): 64.70.Pf, 61.20.Lc, 61.43.Fs

\section{INTRODUCTION}

The static and dynamic behavior of macroscopic systems depends sensitively on the spatial dimension $d$. For example, one-dimensional systems with short-range interactions do not exhibit an equilibrium phase transition. In two dimensions there is no long-range order if the ground state exhibits a spontaneously broken continuous symmetry and Anderson localization occurs for almost all eigenstates of a disordered system for $d=1$ and $d=2$, but not in $d=3$, if the disorder is small. Critical exponents at continuous phase transitions depend on dimensionality. Concerning dynamical features it is known, for instance, that the velocity autocorrelation function of a liquid exhibits a long-time tail proportional to $t^{-d / 2}$. Consequently, the diffusion constant is infinite for $d \leqslant 2$. These few examples demonstrate the high sensitivity of various physical properties of the dimension $d$.

Let us consider a liquid in $d=3$. If crystallization can be bypassed, a liquid undergoes a structural glass transition. Although not all features of this transition are completely understood, recently significant progress has been made concerning its microscopic understanding. Following many decades of several phenomenological descriptions with less predictive power, the mode coupling approach introduced in 1984 by Bengtzelius, Götze, and Sjölander [1] has led to a microscopic theory of the structural glass transition.

This theory, called mode coupling theory (MCT), has been discussed theoretically in great detail by Götze and coworkers (see Ref. [2] for a review). Its numerous predictions were largely successfully checked by experiments and simulations $[3,4]$. The main prediction of MCT is the existence of

*Electronic address: rschill@uni-mainz.de a dynamical glass transition at which the dynamics changes from ergodic to nonergodic behavior. Thermodynamic (equilibrium) quantities-e.g., the isothermal compressibility and structural ones like the static structure factor $S(q)$-do not become singular at the glass transition singularity of MCT. Hence, the MCT glass transition is of pure dynamical nature. It can be smeared-out by additional relaxation channels and then marks a crossover [2,3].

A microscopic theory predicting a structural glass transition with pure thermodynamic origin was derived by Mézard and Parisi [5]. Their replica theory is a first-principles approach which yields a so-called Kauzmann temperature $T_{K}$ at which the configurational entropy vanishes. $T_{K}$ is below $T_{c}$, the MCT glass transition temperature. In low dimensions, $T_{K}$ may mark a crossover [6]. For a review of both microscopic theories as well as phenomenological approaches to the structural glass transition the reader may consult Ref. [7].

An important question is now, what is the dependence of the structural glass transition on the spatial dimensionality? This question has already been asked by several researchers some time ago. Before we come to a short review of this work, let us consider monodisperse hard spheres and hard disks in $d=3$ and $d=2$, respectively.

The most dense packing of four hard spheres corresponds to a regular tetrahedron. However, three-dimensional space cannot be covered completely by regular tetrahedra, without overlapping. This kind of geometrical frustration is absent in two dimensions. The densely packed configuration of three hard disks corresponds to an equilateral triangle. Since the two-dimensional plane can be tiled completely without overlap by equilateral triangles, there is no frustration. Therefore one may be tempted to conclude that there is no structural glass transition in two dimensions. However, the link between frustration and glass transition has proven subtle. Ex- 
periments [8] and simulations $[9,10]$ of monodisperse hard spheres in three dimensions have shown crystallization. Hence, the existence of geometric frustration is not sufficient for glass formation. What one needs is bidispersity or polydispersity.

Santen and Krauth have performed a Monte Carlo (MC) simulation of a two-dimensional system of polydisperse hard disks. The polydispersity has been quantified by a parameter $\varepsilon^{\mathrm{pol}}$. Their results clearly demonstrate (i) the absence of a thermodynamic glass transition and (ii) the existence of a dynamic glass transition at a critical packing fraction $\varphi_{c}\left(\varepsilon^{\mathrm{pol}}\right)$. The kinetic glass transition shifted outside the region of crystallization for $\varepsilon^{\mathrm{pol}} \geqslant \varepsilon_{\min }^{\mathrm{pol}} \approx 10 \%[11,12]$. Furthermore, the diffusivity was found to be consistent with the MCT result $[2,3]$

$$
D \sim\left(\varphi_{c}-\varphi\right)^{\gamma}, \quad \varphi \leqslant \varphi_{c},
$$

where $\gamma \approx 2.4$ and $\varphi_{c}^{\mathrm{sim}} \approx 0.80$. This critical value agrees with what has been found for a related system by Doliwa and Heuer (see Fig. 2 in Ref. [13]). The absence of a thermodynamic glass transition has been strengthened recently by Donev et al. [14] for a binary hard-disk mixture.

There are a few investigations of glass formation in twodimensional systems with soft potentials. Lançon and Chaudhari [15] studied a binary system with modified Johnson potential. They found that the structural relaxation time seems to diverge when approaching a critical temperature. Similar behavior was observed by Ranganathan [16] for a monodisperse Lennard-Jones systems and by Perera and Harrowell [17] for a binary mixture of soft disks with a $1 / r^{12}$ potential. It is surprising that the intermediate self-scattering function $S^{(s)}(q, t)$ of the monodisperse system [16] exhibits strong stretching, one of the characteristics of glassy dynamics. However, $S^{(s)}(q, t)$ does not produce a well-pronounced plateau [16] under an increase of the density; i.e., the cage effect does not become strong enough. This is quite different to the binary system [17]. $S^{(s)}(q, t)$ develops a two-step relaxation process upon supercooling with a well-pronounced plateau over four to five decades in time, at lower temperatures. This behavior is qualitatively identical to that found for, e.g., the Lennard-Jones mixture investigated and analyzed in the framework of MCT by Kob and Anderson [18]. The authors of Refs. [11-17] conclude that there is a structural glass transition in two dimensions. Their conclusion is supported by recent experiments on colloidal particles with repulsive dipolar interactions in two dimensions [19]. Since these simulational and experimental findings strongly resemble the MCT predictions obtained for $d=3$, it is important to apply MCT to two-dimensional liquids. MCT has been applied to the two-dimensional Lorentz model of overlapping hard disks [20] and a charged Bose gas with quenched disorder and logarithmic interactions at zero temperature [21], but to our best knowledge not to a twodimensional liquid-glass problem with self-generated disorder. To accomplish this is the main motivation of the present contribution.

The outline of our paper is as follows. The MCT equations for arbitrary dimensions and the major predictions of MCT will be presented in Sec. II. The theory requires as only input the static structure factor, which is computed in Sec. III. In Sec. IV we apply MCT to a two-dimensional system of monodisperse hard disks and will demonstrate that there is a dynamic glass transition. The dynamic behavior close to that transition is qualitatively identical to that of monodisperse hard spheres in three dimensions. It will also be shown that the MCT result for the time-dependent mean-squared displacement describes the experimental result of Ref. [19] for both species rather satisfactorily over four decades in time. The final section V contains a short summary and some conclusions.

\section{MODE COUPLING EQUATIONS}

In this section we will shortly review the MCT equations for the collective and tagged particle correlator of density fluctuations of a one-component liquid and will present the properties of their solution close to the glass transition singularity. The only dependence on dimension $d$ comes through the integrations element $(2 \pi)^{-d} d^{d} k \sim(2 \pi)^{-d} k^{d-1} d k$ which appears in the memory kernels.

MCT provides equations of motion for the normalized intermediate scattering function $\phi_{q}(t)$ and the tagged particle correlator $\phi_{q}^{(s)}(t)$. The mathematical structure of these equations does not depend on $d$. For Brownian dynamics which is appropriate for colloidal systems they read for $d$ arbitrary

$$
\gamma_{q} \dot{\phi}_{q}(t)+\phi_{q}(t)+\int_{0}^{t} d t^{\prime} m_{q}\left(t-t^{\prime}\right) \dot{\phi}_{q}\left(t^{\prime}\right)=0,
$$

with the memory kernel $m_{q}(t)$ containing fluctuating stresses and playing the role of a generalized friction coefficient. It arises because the density fluctuations captured in $\phi_{q}(t)$ are affected by all other modes in the system. In MCT, one assumes that the dominating contributions at long times are given by density pair fluctuations and approximates (in the thermodynamic limit)

$$
m_{q}(t) \equiv \mathcal{F}_{q}\left[\phi_{k}(t)\right]=\int \frac{d^{d} k}{(2 \pi)^{d}} V(\mathbf{q}, \mathbf{k}, \mathbf{p}) \phi_{k}(t) \phi_{p}(t) .
$$

The vertices express the overlap of fluctuating stresses with the pair density modes and are uniquely determined by the equilibrium structure

$$
V(\mathbf{q}, \mathbf{k}, \mathbf{p})=\frac{n}{2} \frac{S_{q} S_{k} S_{p}}{q^{4}}\left[\mathbf{q} \cdot \mathbf{k} c_{k}+\mathbf{q} \cdot \mathbf{p} c_{p}\right]^{2} \delta(\mathbf{q}-\mathbf{k}-\mathbf{p}),
$$

where $n$ is the number of particles per $d$-dimensional volume, $S_{q}$ the static structure factor, and $c_{q}$ the direct correlation function related to $S_{q}$ by the Ornstein-Zernike equation. $\gamma_{q}$ is a characteristic microscopic time scale. The reader should note that the vertices, Eq. (3), have been approximated by neglecting static three-point correlations.

The corresponding equations for the tagged-particle correlator and $d$ arbitrary are of the same form

$$
\gamma_{q}^{(s)} \dot{\phi}_{q}^{(s)}(t)+\phi_{q}^{(s)}(t)+\int_{0}^{t} d t^{\prime} m_{q}^{(s)}\left(t-t^{\prime}\right) \dot{\phi}_{q}^{(s)}\left(t^{\prime}\right)=0,
$$

with 
$m_{q}^{(s)}(t) \equiv \mathcal{F}_{q}^{(s)}\left[\phi_{k}(t), \phi_{k}^{(s)}(t)\right]=\int \frac{d^{d} k}{(2 \pi)^{d}} V^{(s)}(\mathbf{q}, \mathbf{k}, \mathbf{p}) \phi_{k}(t) \phi_{p}^{(s)}(t)$

and

$$
V^{(s)}(\mathbf{q}, \mathbf{k}, \mathbf{p})=n S_{k} q^{-4}(\mathbf{q} \cdot \mathbf{k})^{2}\left(c_{k}^{(s)}\right)^{2} \delta(\mathbf{q}-\mathbf{k}-\mathbf{p}) .
$$

$c_{k}^{(s)}=\left\langle\rho_{\mathbf{q}}^{(s) *} \rho_{\mathbf{q}}\right\rangle /\left(n S_{q}\right)$ is the tagged-particle direct correlation function. If the tagged particle is one of the liquid's particles, it is $c_{k}^{(s)}=c_{k}$.

The static correlation functions $S_{k}, S_{p}, S_{q}, c_{k}$, and $c_{p}$ and the correlators $\phi_{k}(t), \phi_{k}^{(s)}(t), \phi_{p}(t)$, and $\phi_{p}^{(s)}(t)$ depend on $q=|\mathbf{q}|, k=|\mathbf{k}|$, and $p=|\mathbf{p}|$ only, due to the isotropy of the liquid and glass phase. Therefore the $d$-dimensional integrals in Eqs. (2) and (5) can be reduced to a twofold integral over $k$ and $p$. This will make explicit the $d$ dependence of the vertices. As a result one obtains, similarly to the case in $d=3[1]$,

$$
\begin{aligned}
\mathcal{F}_{q}\left[\phi_{k}(t)\right]= & n \frac{\Omega_{d-1}}{(4 \pi)^{d}} \frac{S_{q}}{q^{d+2}} \int_{0}^{\infty} d k \int_{|q-k|}^{q+k} d p \\
& \times \frac{k p S_{k} S_{p}}{\left[4 q^{2} k^{2}-\left(q^{2}+k^{2}-p^{2}\right)^{2}\right]^{(3-d) / 2}}\left[\left(q^{2}+k^{2}-p^{2}\right) c_{k}\right. \\
& \left.+\left(q^{2}-k^{2}+p^{2}\right) c_{p}\right]^{2} \phi_{k}(t) \phi_{p}(t)
\end{aligned}
$$

and

$$
\begin{aligned}
\mathcal{F}_{q}^{(s)}\left[\phi_{k}(t), \phi_{k}^{(s)}(t)\right]= & 2 n \frac{\Omega_{d-1}}{(4 \pi)^{d}} \frac{1}{q^{d+2}} \int_{0}^{\infty} d k \int_{|q-k|}^{q+k} d p \\
& \times \frac{k p S_{k}}{\left[4 q^{2} k^{2}-\left(q^{2}+k^{2}-p^{2}\right)^{2}\right]^{(3-d) / 2}} \\
& \times\left[\left(q^{2}+k^{2}-p^{2}\right) c_{k}^{(s)}\right]^{2} \phi_{k}(t) \phi_{p}^{(s)}(t),
\end{aligned}
$$

with

$$
\Omega_{d}=\frac{2 \pi^{d / 2}}{\Gamma\left(\frac{d}{2}\right)},
$$

the well-known result for the surface of a $d$-dimensional unit sphere. $\Gamma(x)$ is the gamma function. Note that Eq. (9) yields [with $\left.\Gamma\left(\frac{1}{2}\right)=\pi^{1 / 2}\right]$

$$
\Omega_{1}=2,
$$

which is consistent with the fact that the "one-dimensional unit sphere" is an interval of length two with a "surface" consisting of two points.

The behavior for $q \rightarrow 0$ of both functionals can be obtained by a Taylor expansion of $\int_{k-q}^{k+q} d p(\cdots)$. Although straightforward it is rather tedious. Alternatively, one can start directly from Eqs. (2) and (5), in order to obtain for $q$ $\rightarrow 0$

$$
\begin{aligned}
\mathcal{F}_{q}\left[\phi_{k}(t)\right] \rightarrow & \frac{n}{2} \frac{\Omega_{d}}{2 \pi^{d}} S_{0} \int_{0}^{\infty} d k k^{d-1} S_{k}^{2}\left[c_{k}^{2}+\frac{2}{d} k c_{k} c_{k}^{\prime}\right. \\
& \left.+\frac{3}{d(d+2)} k^{2} c_{k}^{\prime 2}\right]\left[\phi_{k}(t)\right]^{2}+O(q)
\end{aligned}
$$

and

$$
\begin{aligned}
\mathcal{F}_{q}^{(s)}\left[\phi_{k}(t), \phi_{k}^{(s)}(t)\right] \rightarrow & n \frac{\Omega_{d}}{d(2 \pi)^{d}} \frac{1}{q^{2}} \int_{0}^{\infty} d k k^{d+1} S_{k}\left(c_{k}^{(s)}\right)^{2} \\
& \times \phi_{k}(t) \phi_{k}^{(s)}(t)+O(1 / q) .
\end{aligned}
$$

Note that $\mathcal{F}_{0}\left[\phi_{k}(t)\right]$ exists, whereas $\mathcal{F}_{q}^{(s)}\left[\phi_{k}(t), \phi_{k}^{(s)}(t)\right]$ diverges like $q^{-2}$. This divergence is related to the absence of momentum conservation for the tagged particle.

Taking $d=3$ in Eqs. (7), (8), (11), and (12), one arrives at the well-known representations of the memory kernel for finite $q$ and $q \rightarrow 0[1,22,23]$. Note that Refs. [22,23] already present the integrals in Eqs. (7) and (8) in discretized form.

With knowledge of the number density, of the static correlators $S_{q}, c_{q}$, and $c_{q}^{(s)}$ as functions of the thermodynamic variables, and of $\gamma_{q}$ and $\gamma_{q}^{(s)}$ one can solve Eqs. (1) and (4) for initial conditions $\phi_{q}(0)=1$ and $\phi_{q}^{(s)}(0)=1$. There exist several quantities which characterize the solutions. These quantities can be found in Refs. [2,22,23]. In order to keep our presentation self-contained as much as reasonable we discuss those for which results will be reported in the next section. We start with the glass transition singularity. At the glass transition the nonergodicity parameters (NEPs)

$$
f_{q}=\lim _{t \rightarrow \infty} \phi_{q}(t)
$$

change discontinuously from zero to a positive nonzero value, smaller or equal to 1 . The corresponding quantity

$$
f_{q}^{(s)}=\lim _{t \rightarrow \infty} \phi_{q}^{(s)}(t)
$$

can change discontinuously at the same point or in a continuous fashion at higher densities or lower temperatures. Both NEPs fulfill the nonlinear algebraic equations (2)

$$
\frac{f_{q}}{1-f_{q}}=\mathcal{F}_{q}\left[f_{k}\right], \quad \frac{f_{q}^{(s)}}{1-f_{q}^{(s)}}=\mathcal{F}_{q}^{(s)}\left[f_{k}, f_{k}^{(s)}\right] .
$$

$f_{q}^{c}$ and $f_{q}^{(s) c}$ are the NEP at the critical point-e.g., at $n=n_{c}$. Since we will apply MCT to $d$-dimensional hard spheres with diameter $2 R$, we use in the following the packing fraction $\varphi=n \Omega_{d-1}(R)^{d} / d$. Above, but close to $\varphi=\varphi_{c}$-i.e., for $0<\varepsilon \equiv\left(\varphi-\varphi_{c}\right) / \varphi_{c} \ll 1$-it is

$$
f_{q}=f_{q}^{c}+h_{q}\left[\sqrt{\sigma /(1-\lambda)}+\sigma\left(\bar{K}_{q}+\kappa\right) / \sqrt{1-\lambda}\right],
$$

with the critical amplitude

$$
h_{q}=\left(1-f_{q}^{c}\right)^{2} e_{q}^{c},
$$

the separation parameter

$$
\sigma(\varepsilon)=C \varepsilon+O\left(\varepsilon^{2}\right)
$$

and the so-called exponent parameter $\lambda$, which obeys $0<\lambda<1$. The second term on the right-hand side of Eq. (15) 
is the leading asymptotic result for $f_{q}$, and $\bar{K}_{q}+\kappa$ yields the next-to-leading order correction. $C, \lambda, e_{q}^{c}$, and $\bar{K}_{q}+\kappa$ follow from $\mathcal{F}_{q}\left[f_{k}\right]$ and its derivatives with respect to $f_{k}$ at $\varphi_{c}$. Particularly, $e_{q}^{c}$ is the right eigenvector $e_{q}$ belonging to the largest eigenvalue $E_{\max }(\varphi)$ of the stability matrix $\left(\partial \mathcal{F}_{q}\left[f_{k}\right] / \partial f_{k}\right)$ at the critical point. $E_{\max }(\varphi)$ is not degenerate since the stability matrix is non-negative and irreducible. At the critical point the maximum eigenvalue becomes 1 ; i.e., $\varphi_{c}$ can be determined from the condition $E_{\max }\left(\varphi_{c}\right)=1$.

At the critical point $\phi_{q}(t)$ decays to the plateau value $f_{q}^{c}$, $0<f_{q}^{c}<1$. Its time dependence is given by

$$
\phi_{q}^{c}(t)=f_{q}^{c}+h_{q}\left(t / t_{0}\right)^{-a}\left\{1+\left[K_{q}+\kappa(a)\right]\left(t / t_{0}\right)^{-a}\right\}, \quad t \gg t_{0} .
$$

$K_{q}$ (not to be confused with $\bar{K}_{q}$ ) and $\kappa(x)$ are again determined by $\mathcal{F}_{q}\left[f_{k}\right]$ and its derivatives at $\varphi=\varphi_{c}$. They are a measure of the next-to-leading order contribution with respect to the leading asymptotic result

$$
\phi_{q}^{c}(t)=f_{q}^{c}+h_{q}\left(t / t_{0}\right)^{-a}, \quad t \gg t_{0},
$$

the critical law. This critical decay occurs on a time scale $t$ much larger than a typical microscopic time $t_{0}$. The exponent $a$ is determined by $\lambda$, only.

For $\varepsilon<0$ two $\sigma$-dependent, divergent time scales exist:

$$
t_{\sigma}=t_{0}|\varepsilon|^{-1 / 2 a}, \quad \varepsilon><0,
$$

and

$$
t_{\sigma}^{\prime}=t_{0}^{\prime}|\varepsilon|^{-\gamma}, \quad \varepsilon<0,
$$

with $\gamma=\frac{1}{2 a}+\frac{1}{2 b}$ and $t_{0}^{\prime}=t_{0} / B^{1 / b}$, where $B$ is a constant. The so-called von Schweidler exponent $b$ follows from $\lambda$ only. $\phi_{q}(t)$ exhibits a two-step relaxation. The relaxation for $t / t_{\sigma} \ll 1$ to the critical plateau value $f_{q}^{c}$ follows from Eq. (18) by replacing $\left(t / t_{0}\right)$ through $\left(t / t_{\sigma}\right)$, and the decay from that plateau to zero is initiated by the von Schweidler law for $t_{\sigma} \ll t \ll t_{\sigma}^{\prime}$ :

$$
\phi_{q}(t)=f_{q}^{c}-h_{q}\left(t / t_{\sigma}^{\prime}\right)^{b}\left\{1-\left[K_{q}+\kappa(-b)\right]\left(t / t_{\sigma}^{\prime}\right)^{b}\right\} .
$$

$K_{q}+\kappa(-b)$ determines again the next-to-leading-order contribution.

For $\varepsilon>0$ there is a single relaxation process only. $\phi_{q}(t)$ relaxes for $t / t_{\sigma} \ll 1$ like for $\varepsilon<0$, and finally the plateau value $f_{q}$ is reached by an exponentially long time decay.

For $\varepsilon<0$, the final or $\alpha$-relaxation process describes the decay of the correlators from the plateau $f_{q}^{c}$ down to zero. Asymptotically close to the transition, the functional form of the $\alpha$ process is given by a master function $\widetilde{\phi}_{q}(\widetilde{t})$ of the rescaled time $\tilde{t}=t / t_{\sigma}^{\prime}$ via

$$
\phi_{q}(t)=\widetilde{\phi}_{q}(\widetilde{t})+\varepsilon \widetilde{\phi}_{q}^{(2)}(\widetilde{t})+O\left(\varepsilon^{2}\right) \text { for } \varepsilon \rightarrow 0-.
$$

The master function $\widetilde{\phi}_{q}$ obeys an equation similar to Eq. (1) with vertices evaluated right at the critical point, $\varepsilon=0$. Thus it does not depend on separation $\varepsilon$ and control parameters, and Eq. (22) expresses the often observed "(timetemperature) superposition principle" $[2,3]$. The von Schweidler series, Eq. (21), gives the short-time behavior of $\widetilde{\phi}_{q}$ for $\tilde{t} \rightarrow 0$, and the corresponding result for the correction is $\widetilde{\phi}_{q}^{(2)}(\tilde{t} \rightarrow 0) \rightarrow h_{q} B_{1} C \tilde{t}^{-b}$, with $C$ from Eq. (17) and $B_{1}$ a known constant.

Similar leading-order and next-to-leading-order contributions can be derived for the tagged-particle correlator $\phi_{q}^{(s)}(t)$ and, e.g., the mean-squared displacement $\delta r^{2}(t)=\left\langle[\mathbf{r}(t)-\mathbf{r}(0)]^{2}\right\rangle[23]$. Since

$$
\delta r^{2}(t)=\lim _{q \rightarrow 0} \frac{2 d}{q^{2}}\left[1-\phi_{q}^{(s)}(t)\right],
$$

the long-wave limit of Eq. (4) yields after integration with respect to $t$,

$$
\delta r^{2}(t)+D_{0}^{(s)} \int_{0}^{t} d t^{\prime} \tilde{m}_{0}^{(s)}\left(t-t^{\prime}\right) \delta r^{2}\left(t^{\prime}\right)=2 d D_{0}^{(s)} t,
$$

where the memory kernel $\widetilde{m}_{0}^{(s)}(t)$ follows from Eqs. (5) and (12):

$$
\begin{aligned}
\widetilde{m}_{0}^{(s)}(t) & \equiv \lim _{q \rightarrow 0} q^{2} \mathcal{F}_{q}^{(s)}\left[\phi_{k}(t), \phi_{k}^{(s)}(t)\right] \\
& =n \frac{\Omega_{d-1}}{d(2 \pi)^{d}} \int_{0}^{\infty} d k k^{d+1} S_{k}\left(c_{k}^{(s)}\right)^{2} \phi_{k}(t) \phi_{k}^{(s)}(t) .
\end{aligned}
$$

Furthermore, we have used

$$
\gamma_{q}^{(s)}=1 /\left(D_{0}^{(s)} q^{2}\right),
$$

with $D_{0}^{(s)}$ the short-time diffusion constant of the tagged particle. Equations. (13b) and (23) imply that the long-time limit of $\delta r^{2}(t)$,

$$
\lim _{t \rightarrow \infty} \delta r^{2}(t)=2 d r_{s}^{2},
$$

is related to the tagged particle's localization length $r_{s}$ given by

$$
r_{s}^{2}=\lim _{q \rightarrow 0} \frac{1-f_{q}^{(s)}}{q^{2}} .
$$

In the liquid phase where $f_{q}^{(s)}=0$, Eq. (27) gives $r_{s}=\infty$-i.e., the particle is delocalized-while $r_{s}$ becomes finite at the glass transition.

Besides the correlators $\phi_{q}(t)$ and $\phi_{q}^{(s)}(t)$ one can also study the corresponding susceptibilities $\chi_{q}(\omega)$ and $\chi_{q}^{(s)}(\omega)$, respectively. Similar asymptotic laws and next-to-leadingorder corrections exist for them [22,23]. Independent of whether the correlators or their susceptibilities are considered, the dependence on $d$ of the leading- and next-toleading-order terms enters only via the $d$ dependence of $\mathcal{F}_{q}$ [Eq. (7)] and $\mathcal{F}_{q}^{(s)}$ [Eq. (8)].

\section{STATIC STRUCTURE}

In this section we consider the calculation of accurate equilibrium structural correlation functions for the hard disk system. Within MCT, all information regarding the interparticle interactions is contained in the static structure factor which enters the memory function vertices, Eqs. (3) and (6); 
the interaction potential does not enter explicitly in the MCT equations. Experience with MCT calculations in threedimensional systems has shown that the location of the glass transition somewhat depends on the details of the input structure factor, particularly the height of the main peak. Different approximate theories for the static structure lead to varying values for, e.g., the critical packing fraction $\phi_{c}^{(d=3)}$ [24]. We have therefore considered a number of approximation schemes for the two- dimensional static structure factor in order to obtain the best possible values for the description of the ideal glass transition. The quality of the various approximation schemes is assessed by comparison with computer simulation data. (Monte Carlo simulations of $6 \times 10^{4}$ particles, as well as event-driven molecular dynamics simulations of 1089 particles, were performed, both in the NVT ensemble [26].)

Integral equation theories based on the Ornstein-Zernike (OZ) equation provide a powerful method to calculate the pair correlation functions for a given interaction potential [25]. The $\mathrm{OZ}$ equation is given by

$$
h(r)=c(r)+n \int d^{d} r^{\prime} c\left(r^{\prime}\right) h\left(r-r^{\prime}\right),
$$

where $h(r) \equiv g(r)-1$. This expression must be supplemented by an additional (generally approximate) closure relation between $c(r)$ and $h(r)$. For hard spheres in $d$ dimensions the most widely used closure is the Percus-Yevick(PY) relation $g(r<1)=0, c(r>1)=0$. In odd dimensions the resulting integral equation can be solved analytically for the direct correlation function $c(r)$. In even dimensions there exists no analytic solution and a full numerical solution is required [27]. Efforts have been made to approximate the numerical PY data by analytic forms $[28,29]$ but in all cases these fail to reproduce accurately the detailed structure of the numerical solution at high densities. The formally exact closure to the $\mathrm{OZ}$ equation for systems with pairwise interactions is given by

$$
h(r)=-1+\exp [-\beta u(r)+h(r)-c(r)+B(r)],
$$

where $u(r)$ is the pair potential and $B(r)$ is the bridge function, an intractable function representing the sum of the most highly connected diagrams in the virial expansion. Setting $B(r)=0$ recovers the familiar hypernetted-chain approximation.

The modified-hypernetted-chain (MHNC) approximation is to take $B(r)$ from the PY theory solved at some effective density $n^{*}$, different from the true system density $n$, and to treat this as a variational parameter to ensure thermodynamic consistency between the virial and compressibility routes to the pressure. A detailed description of the MHNC equation can be found in [30]. The steps taken in solving the MHNC equation are the following: (i) numerically solve the PY equation at density $n^{*}$, (ii) use Eq. (30) to find $B(r)$ $\equiv B_{P Y}\left(r ; n^{*}\right)$, (iii) solve Eqs. (29) and (30) with this bridge function, (iv) calculate the pressure from the virial and compressibility equations, and (v) adjust $n^{*}$ until the two pressures are equal. In three dimensions it is generally recognized that the MHNC approximation provides a highly

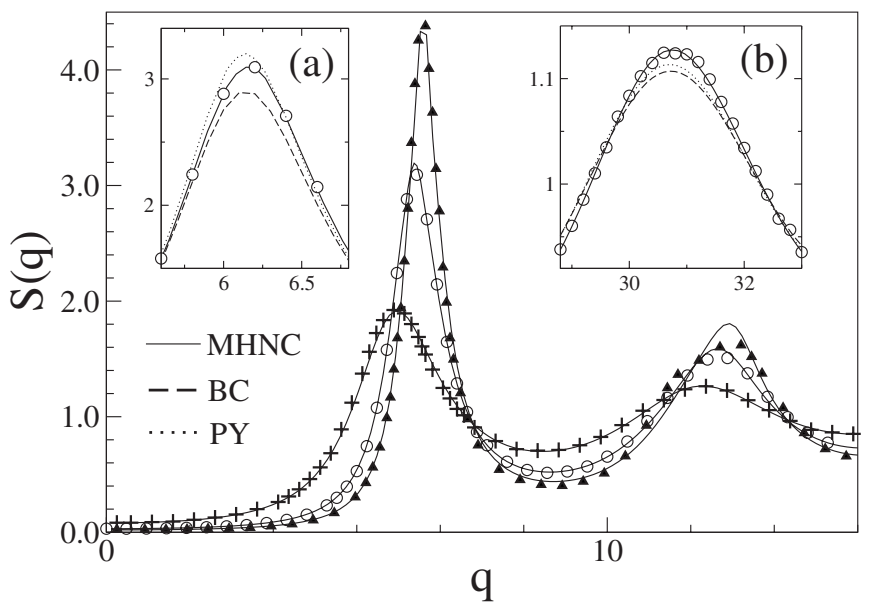

FIG. 1. Comparison between theoretical MHNC structure factors and simulation for hard disks at packing fractions $\phi=0.5(+)$, $0.628(\bigcirc)$, and $0.68(\Delta) ; q$ is given in units of $1 /(2 R)$, the inverse diameter. Inset (a) concentrates on the vicinity of the main peak and gives additional comparison with Baus-Colot and PY theories for packing fraction $\phi=0.628$. Inset (b) demonstrates how the MHNC theory correctly captures the asymptotic behavior for large $q$ values for packing fraction $\phi=0.628$.

accurate description of the pair correlations for the hardsphere fluid, significantly improving upon the PY theory. We find that the same is true in the case of two-dimensional hard disks. At low densities the MHNC $S_{q}$ lies very close to the PY result. As the density increases discrepancies begin to arise, particularly in the region of the main peak, with the MHNC in closer agreement with simulation. Both the MHNC and PY theories are significantly more accurate than the analytical Baus-Colot expression [28]. Figure 1 shows a comparison between the MHNC $S_{q}$ and the simulation results. The level of agreement is very satisfactory, and the MHNC shows clear improvement over the other theories investigated. The only notable deviation from the simulation results is the height and width of the second (third) peak, which is overestimated (underestimated) by the MHNC theory. It is known that upon approaching the crystallization phase boundary (located at $\phi_{F}=0.69$ for hard disks) a shoulder develops on the second peak of the structure factor, a feature which has been interpreted as an indicator of approaching crystallization [31]. The development of the shoulder also suppresses the height of the second peak to some extent and leads to a small shift in the location of the third peak. The MHNC theory, like the PY and all other standard integral equation theories, does not contain information about crystallization and thus predicts fluidlike structure at, and beyond, the freezing transition. While this property leads to some discrepancy with simulation results at high density, it makes such theories ideal for calculating the fluidlike structure factors required as input to the MCT, where we assume crystallization to have been suppressed. We can thus proceed with confidence using MHNC structure factors as input to the MCT.

\section{RESULTS}

In this section we will apply MCT to a two-dimensional (2D) system of monodisperse hard disks with diameter $2 R$. 


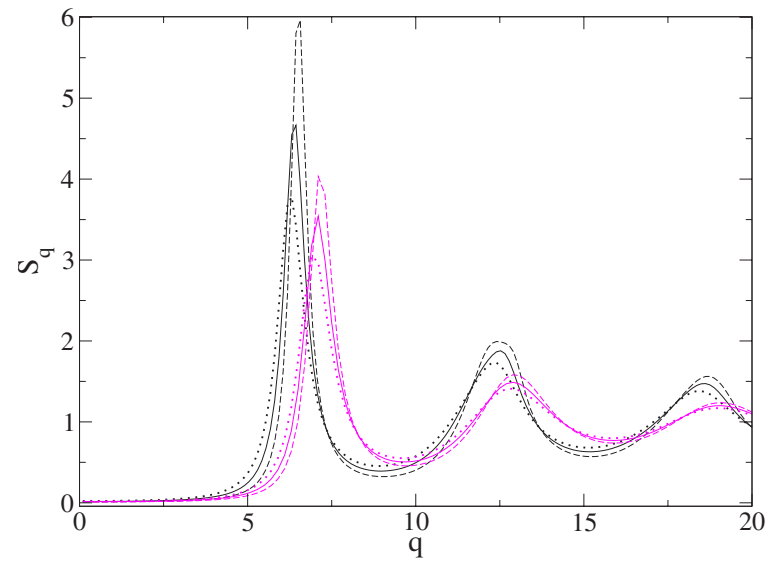

FIG. 2. (Color online) Comparison of 2D MHNC structure factors (black/dark) to 3D PY (magenta/light). The packing fractions correspond to $\varepsilon=0$ (solid lines), $\varepsilon=-10^{-4 / 3}$ (dotted lines), and $\varepsilon=$ $+10^{-4 / 3}$ (dashed lines). The critical packing fractions are 0.697 for $2 \mathrm{D}$ and 0.516 for $3 \mathrm{D}$.

We will solve the MCT equations (1), (4), and (24) and will present results for those quantities discussed in the last section. As input we will use the static structure factors $S_{q}$ obtained from the MHNC approach. This result is presented in Fig. 2 for three different packing fractions close to the glass transition and compared with the corresponding PercusYevick result for hard spheres in $d=3$. For instance, for $\varphi=\varphi_{c}^{d=2}$ and $\varphi=\varphi_{c}^{d=3}$-i.e., for $\varepsilon=0$ - the peaks are more pronounced in $d=2$. In particular the main peak is more narrow and higher for $d=2$ than for $d=3$. The direct correlation function $c_{q}$ follows from the Ornstein-Zernike equation. Because we choose the tagged particle as one of the liquid particles, we have $c_{q}^{(s)}=c_{q}$.

For the friction coefficients in Eqs. (1) and (4) we take $\gamma_{q}=S_{q} /\left(D_{0}^{(s)} q^{2}\right)$ and $\gamma_{q}^{(s)}=1 /\left(D_{0}^{(s)} q^{2}\right)$ [cf. Eq. (28)], and choose as our unit of time $\tau^{(B D)}=D_{0}^{(S)} /\left[10(2 R)^{2}\right]$; in the following all times will be given as rescaled ones, $t / \tau^{(B D)}$. For the numerical solution of MCT equation one has to discretize $q$. A compromise between a fine grid and computation time is required, as the computations scale with number of grid points, $M^{3}$. We choose a grid with $M=250$ grid points and a high- $q$ cutoff of $50 /(2 R)$. A higher cutoff has only a very small effect on the critical packing fraction $\left(<10^{-4}\right)$. The effect of the number of grid points is more sensitive due to the form of the Jacobian of the transformation to bipolar coordinates. In this paper the integration is substituted by a rule that can be called a modified trapezoid rule. The value of the function to be integrated is not taken in the middle of the interval $[n h,(n+1) h]$ but at $(n+0.303) h$. By using this rule one gets the best discrete description of the Jacobian and hence it is used here. The difference in $\varphi$ between $M=500$ and $M=250$ is then $<10^{-3}$, leading to a system close enough to continuum. Then the solution of Eqs. (1), (4), and (24) will be performed by use of a decimation technique [32].

The search for the glass transition singularity can be done either by an iterative solution of the nonlinear equations (14) or by calculation of $E_{\max }(\varphi)$. In this paper a simple bracketing algorithm is used starting from two points where point $A$

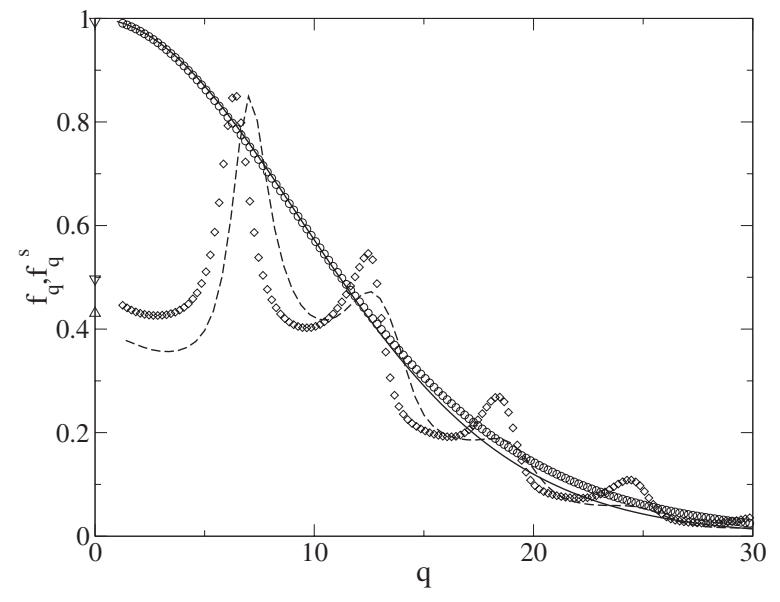

FIG. 3. Nonergodicity parameter of coherent (2D diamond, 3D dashed line) and incoherent (2D circles, 3D solid line) correlators at critical packing fraction $\varphi_{c}^{2 D}=0.697$ and $\varphi_{c}^{3 D}=0.516$. The $q$ values for $q<1$ are not included since they can not be determined accurately for numerical reasons. The $q=0$ results are from the analytic expansions, Eqs. (11) and (12), and are included as triangles.

yields a finite NEP for $q$ near the peak position and point $B$ has $f_{q}=0$. The next point $C$ is taken in the middle of the interval. If it yields finite $f_{q}$ the next point is taken between $A$ and, $C$; if $f_{q}=0$ the other interval $[C, B]$ is taken. This procedure is continued until the critical packing fraction $\varphi_{c}$ is determined to a precision of $10^{-10}$. As a result we have found

$$
\varphi_{c}^{d=2} \cong 0.696810890(317)
$$

which is above the value for $d=3$ [22]:

$$
\varphi_{c}^{d=3} \cong 0.51591213(1)
$$

(The denoted accuracy will be required to reliably compute $\varepsilon$ in the following.) Similar to the three-dimensional system, the collective part and self-part of the density fluctuations become nonergodic at the same critical packing fraction $\varphi_{c}^{d=2}$. The corresponding critical nonergodicity parameters are shown in Fig. 3.

While almost no difference between incoherent NEPs for $d=2$ and $d=3$ can be observed, more pronounced maxima appear in the coherent NEP at higher wave vectors for the lower dimension. Regions of rather abrupt $q$ dependences in $f_{q}$ should be observable experimentally. Two length scales appear to be involved in $f_{q}$. While the average particle distance, connected to the main peak in $S_{q}$, somewhat differs from $d=3$ to $d=2$, the localization length, which dominates the incoherent NEP, is insensitive to dimensionality. The change of $f_{q}$ when stepping down in dimension thus cannot simply be scaled away.

An important observation in the numerical solution of Eq. (14) concerns the convergence of the required integrals. We find here, and for all other integrations performed, that convergence at small and large wave vectors holds. No critical anomalies arise connected with the growth of a static correlation length [33]. We interpret this as indication that the 


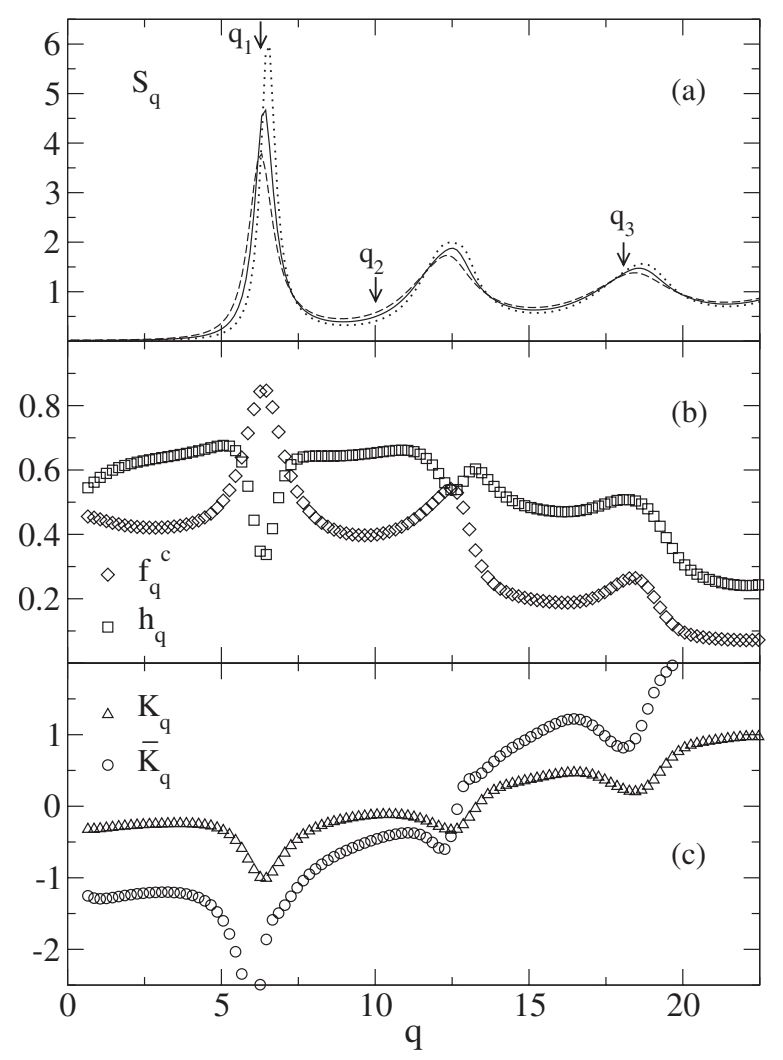

FIG. 4. (a) Structure factor $S_{q}$ as function of wave vector $q$ for $\varphi=\varphi_{c} \approx 0.697$ (solid line), $\varphi \approx 0.729$ (dotted line), and $\varphi \approx 0.664$ (dashed line); the latter correspond to $\varepsilon= \pm 10^{-4 / 3}$. The arrows mark the wave vectors $q_{1}=6.46, q_{2}=10.06$, and $q_{3}=18.26$. (b) Critical NEP $f_{q}^{c}$ (diamonds) and critical amplitude $h_{q}$ (squares). (c) The amplitudes $K_{q}$ (triangles) and $\bar{K}_{q}$ (circles).

MCT glass transition in $d=2$ describes a local phenomenon not affected by long-range correlations, which might sensitively depend on dimensionality.

All wave-vector-dependent structure functions describing the glassy structure and its relaxation ("cage effect") are summarized in Fig. 4. The critical nonergodicity parameter $f_{q}^{c}$ and the critical amplitude $h_{q}$ are depicted in Fig. 4(b) and the next-to-leading-order amplitudes $K_{q}$ and $\bar{K}_{q}$ [cf. Eqs. (15), (18), and (21)] and in Fig. 4(c). The $q$ dependence of the shown quantities follows generally the one of $S_{q}$. Only $h_{q}$ exhibits the opposite variation around the main structure factor peak. Figure 4(a) specifies three typical wave numbers for which results will be discussed below. Comparison with the $d=3$ result (Fig. 2 in Ref. [22]) reveals a qualitatively similar $q$ dependence of $f_{q}^{c}, h_{q}, K_{q}$ and $K_{q}$. Like for $S_{q}$, the $q$ variation of all quantities is more pronounced in $d=2$ than in $d=3$.

As mentioned in Sec. II, the separation parameter $\sigma(\varepsilon)$ can be calculated from $\mathcal{F}_{q}\left[f_{k}\right]$ and its derivatives at $\varphi_{c}$. The result is given in the inset of Fig. 5. The linear term in Eq. (17) describes $\sigma(\varepsilon)$ for $-0.030 \leqslant \varepsilon \leqslant 0.025$ with an accuracy better than $10 \%$. This range is similar to that for the corresponding result for $d=3$ [22] and provides an estimate for the range of validity of the asymptotic expansions. The quality of the leading-order result and the next-to-leading-order con-

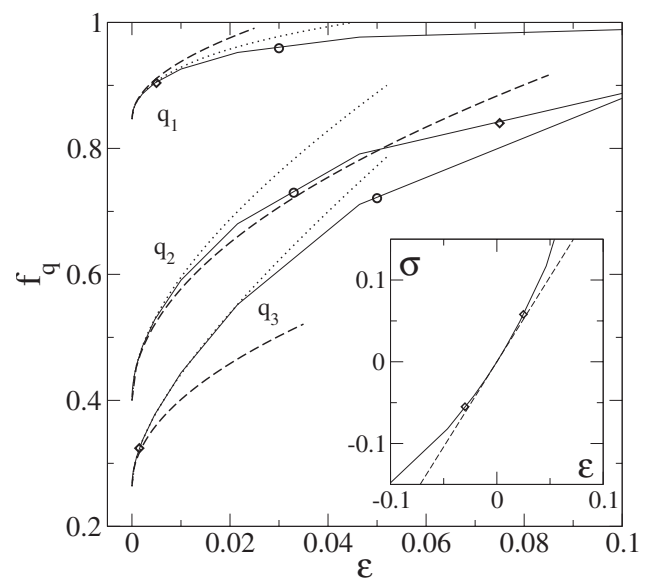

FIG. 5. Nonergodicity parameter $f_{q}$ for $q_{1}=6.46, q_{2}=10.06$, and $q_{3}=18.26$ (solid lines). The leading asymptotes (dashed line) describe $f_{q}-f_{q}^{c}$ within $10 \%$ up to the values marked by diamonds $(0.005,0.075,0.0015)$. The next-to-leading asymptotes (dotted line) are within $10 \%$ for $\varepsilon$ up to the values marked by circles $(0.03$, $0.033,0.05)$. The values for the correction amplitudes are $C=2.08$, $\kappa=1.18, h_{1}=0.337, h_{2}=0.654, h_{3}=0.508, \bar{K}_{1}=-1.86, \bar{K}_{2}=-0.456$, and $\bar{K}_{3}=0.844$. The inset shows the separation parameter $\sigma$ as a function of $\varepsilon$. The dashed line is the linear asymptote $\sigma=C \varepsilon$. The range where the asymptote deviates less than $10 \%$ from $\sigma$ is between the diamonds that are at $\varphi=0.676$ and $\varphi=0.714$.

tribution for $f_{q}$ [cf. Eq. (15)] is demonstrated in Fig. 5 for the three $q$ values $q_{1}, q_{2}$, and $q_{3}$ [Fig. 4(a)]. It is interesting, that for $q_{2}$ the leading asymptote describes $f_{q}(\varepsilon)$ best and for an unexpectedly wide range. This arises because of a cancella-

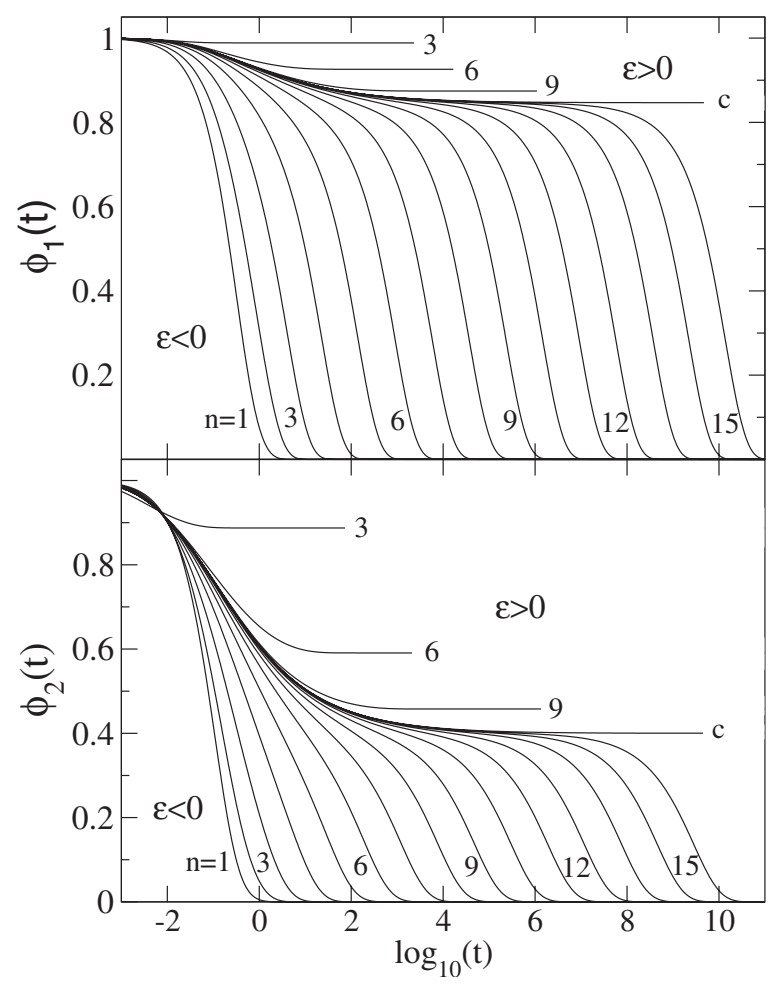

FIG. 6. Coherent correlators $\phi_{1}(t)$ (top) and $\phi_{2}(t)$ (bottom) for different $\varepsilon= \pm 10^{-n / 3}$. Curves are labeled by $n$. 


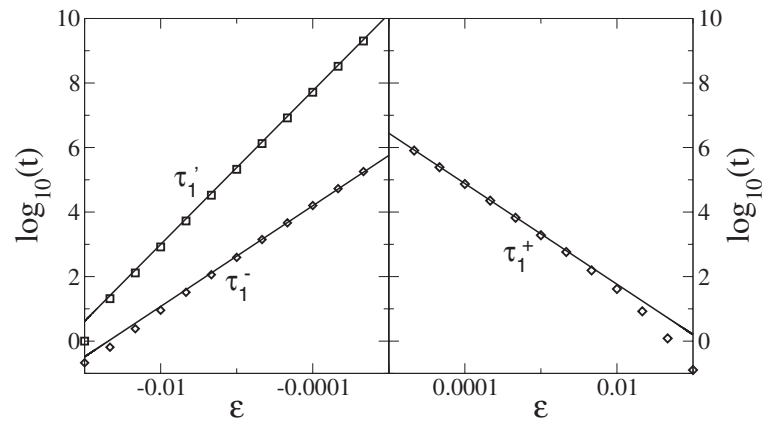

FIG. 7. Timescales $\tau^{ \pm}$for the $\beta$ process and $\tau^{\prime}$ for the $\alpha$ process in the liquid for wave vector $q_{1}$ (in double-logarithmic presentation). The lines are the asymptotic power laws. $\lambda=0.7167, a$ $=0.320, \quad b=0.613, \delta=\frac{1}{2 a}=1.56, \quad c_{+}=0.044, \quad c_{-}=0.009, \quad \gamma=\frac{1}{2 a}+\frac{1}{2 b}$ $=2.38$, and $c^{\prime}=0.0173$. (For the definitions of $c_{ \pm}$and $c^{\prime}$ see Ref. [22].)

tion of higher-order correction terms. For $q_{1}$ and $q_{3}$ the nextto-leading order has to be taken into account already for rather small $\varepsilon$, as expected from the $\sigma(\varepsilon)$ curve. The overall behavior also is quite similar to $d=3$ (cf. Fig. 3 of Ref. [22]).

Now we turn to dynamical features. Figure 6 presents the normalized correlators $\phi_{i}(t) \equiv \phi_{q_{i}}(t)$ for $i=1,2$. The two-step relaxation process for $\varepsilon<0$ becomes obvious for both correlators. Since $f_{q_{2}}^{c}<f_{q_{1}}^{c}$ [cf. Figs. 4(a) and 4(b)] the plateau heights for $\phi_{2}(t)$ are below those for $\phi_{1}(t)$. Again, the $t$ and $\omega$ dependence (the latter is not shown) is in qualitative agreement with the corresponding results in $d=3$ (cf. Figs. 4 and 6 of Ref. [22]). In the following, we will apply the asymptotic expansions from Sec. II to the correlators in order to characterize the long-time dynamics in more detail.

Following Ref. [22] we have calculated typical time scales $\tau_{q}^{ \pm}(\varepsilon><0)$ and $\tau_{q}^{\prime}(\varepsilon<0)$ characterizing the first and second relaxation steps. In the fluid, $\tau^{-}(q)$ marks the crossing of the plateau, $\phi_{q}\left(\tau^{-}(q)\right)=f_{q}^{c}$, and the $\alpha$-relaxation time is defined by $\phi_{q}\left(\tau_{q}^{\prime}\right)=f_{q}^{c} / e$. In the glass, $\tau^{+}(q)$ captures the approach to the long-time plateau, $\phi_{q}\left(\tau_{q}^{+}\right)-f_{q}^{c}=1.001\left(f_{q}-f_{q}^{c}\right)$. Results are shown in Fig. 7 for $q=q_{1}$. The divergence of these relaxation times at $\varepsilon=0$ is described by the asymptotic

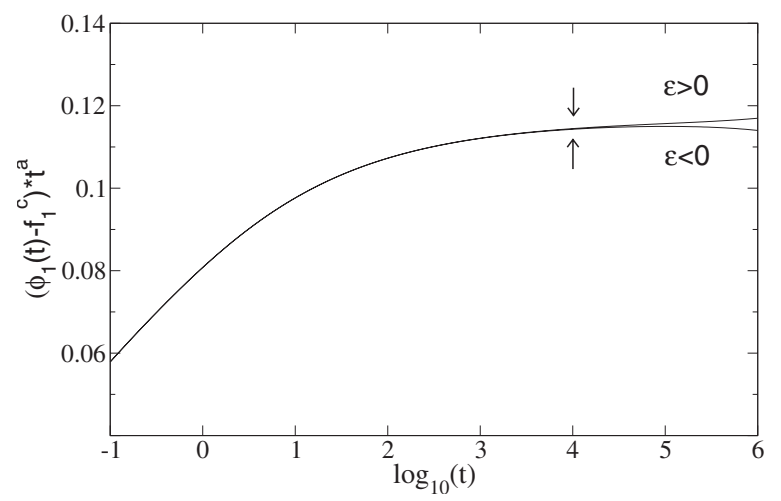

FIG. 8. Determination of $t_{0}$. $\left[\phi_{1}(t)-f_{1}^{c}\right] t^{a}$ is plotted over $\log t$, for glass and liquid curves close to the transition. The position where the plateau is best reached by both curves is marked by arrows. The value found is $0.114 . t_{0}$ is then given by $\left(\frac{0.114}{h_{1}}\right)^{1 / a}$ $=0.034$, with $h_{1}=0.337$ and $a=0.320$.

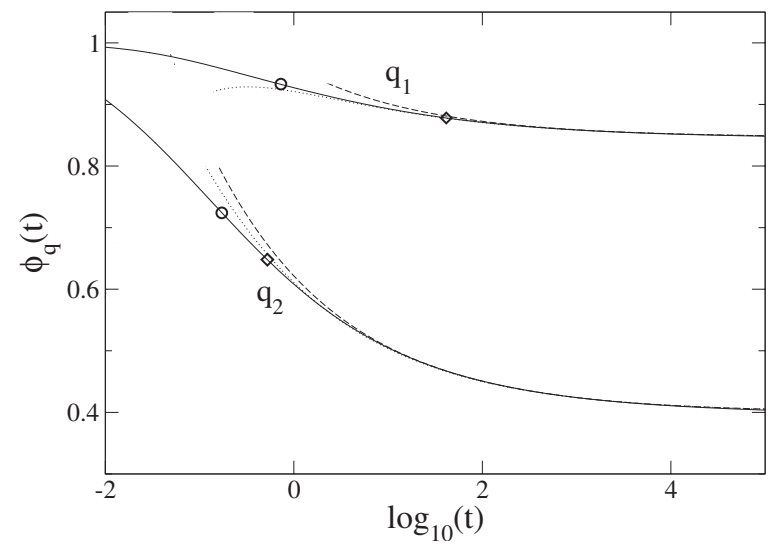

FIG. 9. The critical laws. The leading asymptotes (dashed line) describe the solution within $10 \%$ up to the points marked by diamonds $(41,0.52)$ for $\left(q_{1}, q_{2}\right)$. The next-to-leading results (dotted) are within $10 \%$ up to the circles $(0.73,0.17)$ for $\left(q_{1}, q_{2}\right)$. Correction amplitudes are $\kappa(a)=-0.021, K_{1}=-1.007$, and $K_{2}=-0.120$.

laws, Eqs. (20a) and (20b). Since $\varphi_{c}^{d=2}$ has been determined, one can calculate the exponent parameter $\lambda$. As a result we find $\lambda^{d=2} \cong 0.7167$ which implies $a^{d=2} \cong 0.320, b^{d=2} \cong 0.613$, and $\gamma^{d=2} \cong 2.38$. These values are close to $\lambda^{d=3} \cong 0.735$, $a^{d=3} \cong 0.312, b^{d=3} \cong 0.583$, and $\gamma^{d=3} \cong 2.46$ [22]. Using in Eqs. (20a) and (20b) $a^{d=2}$ and $\gamma^{d=2}$ leads to the asymptotes (solid lines) in Fig. 7.

The microscopic time scale $t_{0}$ entering the critical power law, Eq. (18), can be deduced by plotting $\left[\phi_{q}(t)-f_{q}^{c}\right] t^{a}$ versus $\log t$ for $\varepsilon>0$ and $\varepsilon<0$ (see Fig. 8). The value at which a constant plateau is best reached by both curves is 0.114 $=h_{q} t_{0}^{a}$. With $h_{q_{1}} \cong 0.337$ and $a^{d=2}$ we get $t_{0}=0.034$. The quality of the leading-order result [Eq. (19)] and its next-toleading-order correction [second and third terms in the curly bracket of Eq. (18)] of the critical law is checked in Fig. 9. A similar check for the von Schweidler law (Eq. (21)) is done in Fig. 10. Like for $f_{q}^{c}$, the leading order has a large range of validity for $q=q_{2}$. Note that both time scales of the structural

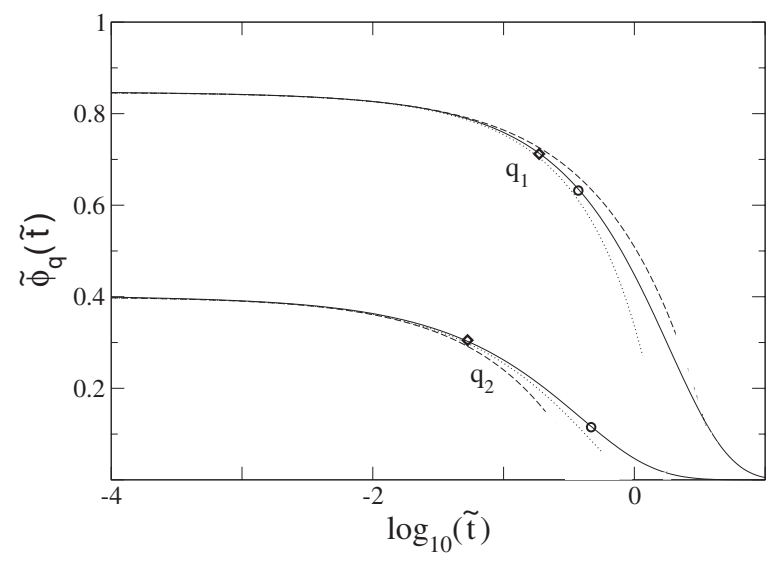

FIG. 10. The von Schweidler law. The leading asymptotes (dashed line) describe the solution within $10 \%$ up to the points marked by diamonds $(0.29,0.053)$ for $\left(q_{1}, q_{2}\right)$. The next-to-leading results (dotted line) are within $10 \%$ up to the circles $(0.37,0.47)$ for $\left(q_{1}, q_{2}\right) \quad\left(b=0.613\right.$ and $\left.t_{\sigma}^{\prime}=7.45 \times 10^{9}\right)$. Correction amplitudes are $\kappa(b)=0.496, K_{1}=-1.007$, and $K_{2}=-0.120$. 


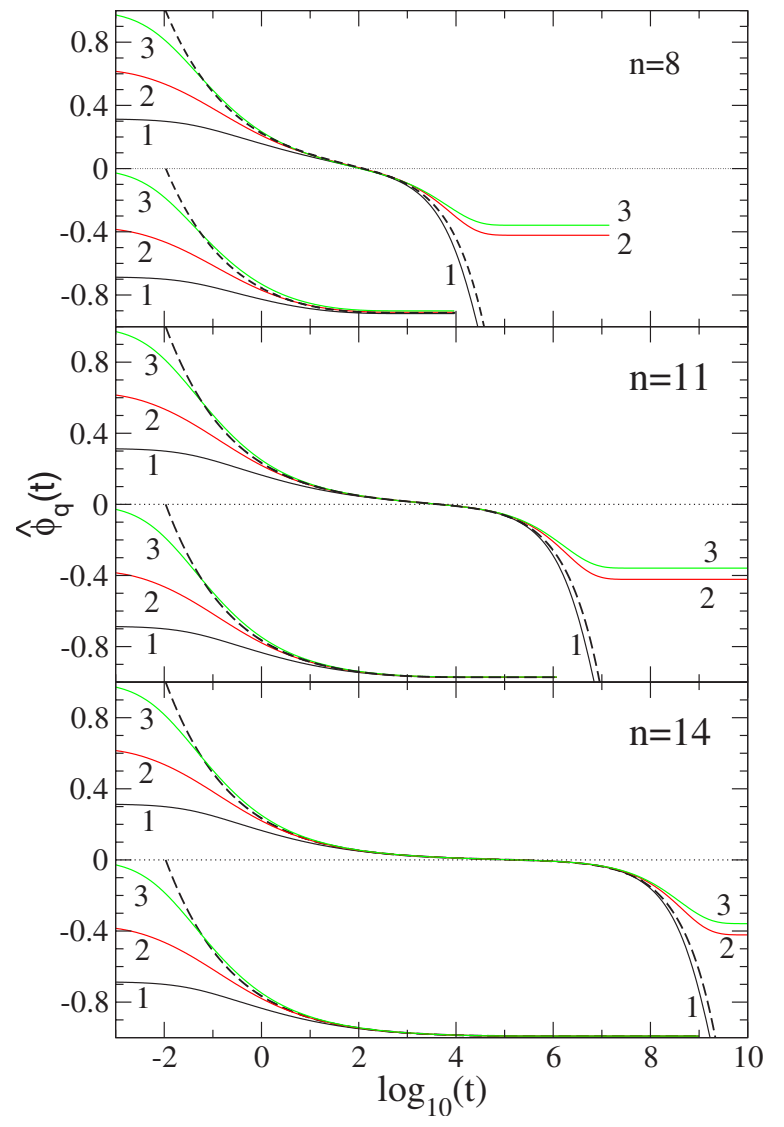

FIG. 11. (Color online) Functions $\hat{\phi}_{q}(t)=\left[\phi_{q}(t)-f_{q}^{c}\right] /\left(h_{q} \sqrt{C}\right)$ for $q_{1}=6.46, q_{2}=10.06$, and $q_{3}=18.26$ for $\varepsilon= \pm 10^{-n / 3}$ for 3 values of $n$ (solid lines). The scaling asymptotes $G(t) / \sqrt{C}$ are shown as dashed lines. The region where the functions for different $q$ collapse onto a master function ( $\beta$ region) increases with decreasing $\varepsilon . C=2.08$; the curves for $\varepsilon>0$ are shifted down by 1 .

relaxation are given by the matching time $t_{0}$ determined in Fig. 8, and by the separation parameter $\varepsilon$. Thus, there remains no adjustable parameter in the test of the von Schweidler law in Fig. 10.

The critical law and the von Schweidler law are the shortand long-time expansions of the so-called $\beta$-master function $g_{ \pm}\left(\hat{t}=t / t_{\sigma}\right)$ for $\varepsilon \gtrless 0$, respectively. $g_{ \pm}(\hat{t})$ describes the first scaling-law regime. The $t$ and $\varepsilon$ dependence of $\hat{\phi}_{q}(t)$ $=\left(\phi_{q}(t)-f_{q}^{c}\right) /\left(h_{q} C\right)$ on the time scale $t_{\sigma}$ is given by $g_{ \pm}(\hat{t})$ for $|\varepsilon| \rightarrow 0$, independent of on $q$ [2]. For the two-dimensional system this property is demonstrated in Fig. 11. We clearly observe that the curves for $q_{1}, q_{2}$, and $q_{3}$ collapse onto a master function with increasing $n-$ i.e., for $\varepsilon \rightarrow 0$. Because of the connection between the $q$ dependences of the correction amplitudes in Eqs. (18) and (21), an ordering scheme exists for the functions $\hat{\phi}_{q}(t)$ in Fig. 11. Their vertical order before and after crossing the plateau needs to coincide; this is obeyed in Fig. 11.

The second scaling-law regime (for $\varepsilon<0$ ) is defined by the rescaled time $\tilde{t}=t / t_{\sigma}^{\prime}$, where $t_{\sigma}^{\prime}$ often is called the $\alpha$-relaxation time and denoted by $\tau$. For $\tilde{t}=\mathcal{O}(1)$, the $t$ and $\varepsilon$ dependence of $\phi_{q}$ is given by the $\alpha$-master function $\widetilde{\phi}_{q}(\widetilde{t})$ [2]. The validity of this second scaling law [Eq. (22)] is

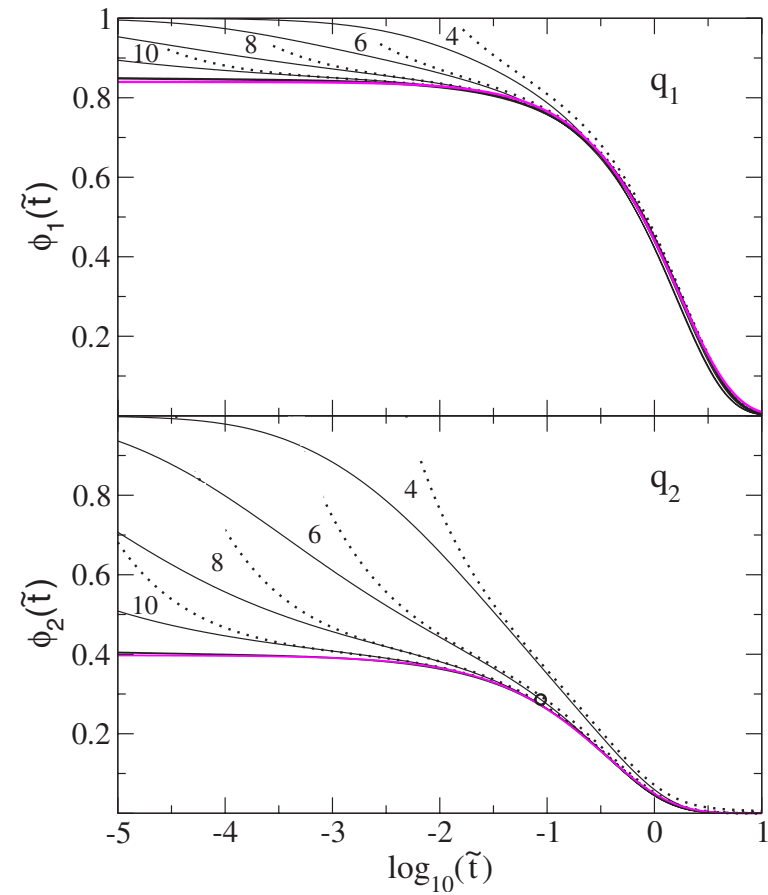

FIG. 12. (Color online) Correlators $\phi_{q}(\widetilde{t})$ for different $\varepsilon=$ $-10^{-n / 3}, n=4,6,8,10$, as function of rescaled time $\tilde{t}=t / t_{\sigma}^{\prime}$ (solid lines). The thick solid line is the $\alpha$-master function $\widetilde{\phi}_{q}(\widetilde{t})$. The dotted lines are the short time parts of leading-plus-next-to-leading approximation for the $\alpha$ correlators $\widetilde{\phi}_{q}(\widetilde{t})+h_{q} B_{1} \sigma \widetilde{t}^{-b}$ according to Eq. (22) with $B_{1}=0.5 /[\Gamma(1-b) \Gamma(1+b)-\lambda]=0.374$. Light (magenta) curves give the Kohlrausch laws fitted to the $\widetilde{\phi}_{q}(\widetilde{t})$ in the range $\log _{10}(\widetilde{t})=[-3.86,2.14]$; the parameters are given in Fig. 13 .

presented in Fig. 12. Approaching $\varepsilon=0$ from below a collapse onto a $q$ dependent master function $\widetilde{\phi}_{q}$ occurs.

For the test of the superposition principle, the $\alpha$-relaxation time was computed using the power law, Eq. (20b), and $t_{0}$ from Fig. 8. At $q_{1}$, the $\alpha$-relaxation time obviously deviates early from the asymptotic power law, since there are intersections of the rescaled correlators. Nevertheless, the range of validity of the $\alpha$-scaling law in Fig. 12 far exceeds the one of the $\beta$-scaling law tested in Fig. 11. This originates from the dependence of the leading corrections on the separation parameter $\varepsilon$. While the corrections to the $\beta$ process are smaller by a factor $\sqrt{\varepsilon}$ only, the relative corrections to the $\alpha$-superposition principle start out in order $\varepsilon$. For example, at $q_{2}$, the $\alpha$ master function $\widetilde{\phi}_{q_{2}}(\widetilde{t})$ describes $68 \%$ of the decay of the final relaxation better than on a $5 \%$ error level at the separation $\varepsilon=-0.01$ (see the circle in Fig. 12), while in Fig. 11 for the test of the $\beta$-scaling law smaller $\varepsilon$ are required.

The shape of the $\alpha$-relaxation process-viz, its master functions $\widetilde{\phi}_{q}(\widetilde{t})$-often is described by a Kohlrausch law

$$
\widetilde{\phi}_{q}(\widetilde{t}) \approx A_{q} \exp \left\{-\left(\frac{\tilde{t}}{\widetilde{\tau}_{q}}\right)^{\beta_{q}}\right\},
$$

where a possible dependence of the parameters on wave vector $q$ is taken into account. The von Schweidler expansion of 


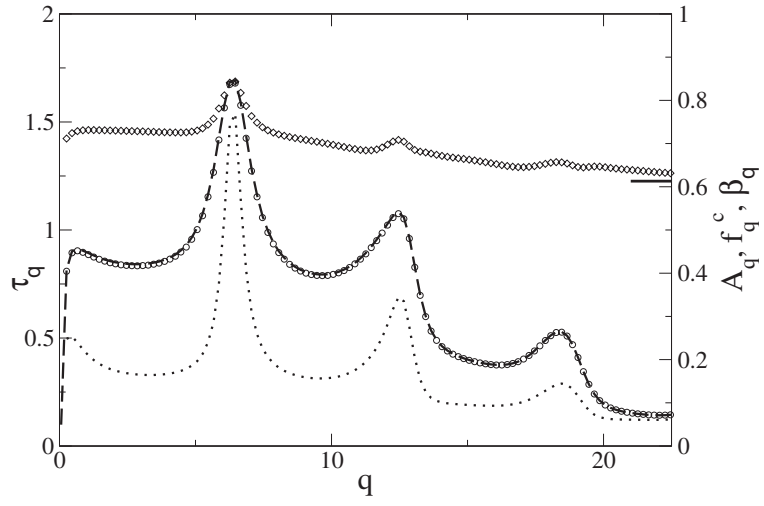

FIG. 13. Kohlrausch fit parameters of a least-squares fit of Eq. (31) to the MCT $\alpha$-master functions $\widetilde{\phi}_{q}(\widetilde{t})$ on the logarithmic time axis in the interval $\log _{10}(\widetilde{t})=[-3.86,2.14]$. $A_{q}$ (circles) is quite close to $f_{q}^{c}$ (thick dashed). The Kohlrausch exponent $\beta_{q}$ (diamonds) converges to the von Schweidler exponent $b=0.613$ (thick line) for high $q$ values. The times $\tilde{\tau}_{q}$ are shown by a dotted line.

the $\alpha$ process, Eq. (21), immediately shows that the $\widetilde{\phi}_{q}(\widetilde{t})$ exhibit stretching - viz., do not decay via simple exponential relaxation, but result from a broad distribution of relaxation times. Numerical solutions of the MCT equations in $d=3$ have shown that, e.g., for hard spheres, the Kohlrausch law provides a good overall fit to the master functions for all wave vectors albeit with noticeable deviations at short rescaled times and with $q$-dependent parameters [34]. Interestingly least-squares fits of Eq. (31) to the (final part of the) $\alpha$-master functions for $\tilde{t}>10^{-3.86}$ yield remarkably close agreement of the $d=2$ curves with Kohlrausch laws. This is shown for two wave vectors in Fig. 12 and can be learned from the fit parameters presented in Fig. 13. The amplitude $A_{q}$ of the Kohlrausch law closely follows the critical NEP, which gives the (true) amplitude of the $\alpha$ process, and the Kohlrausch stretching exponent $\beta_{q}$ varies little with wave vectors and is quite close to its large- $q$ limit given by the von Schweidler exponent $b$. Within MCT, the Kohlrausch law can be derived as limiting law when an increasing number of

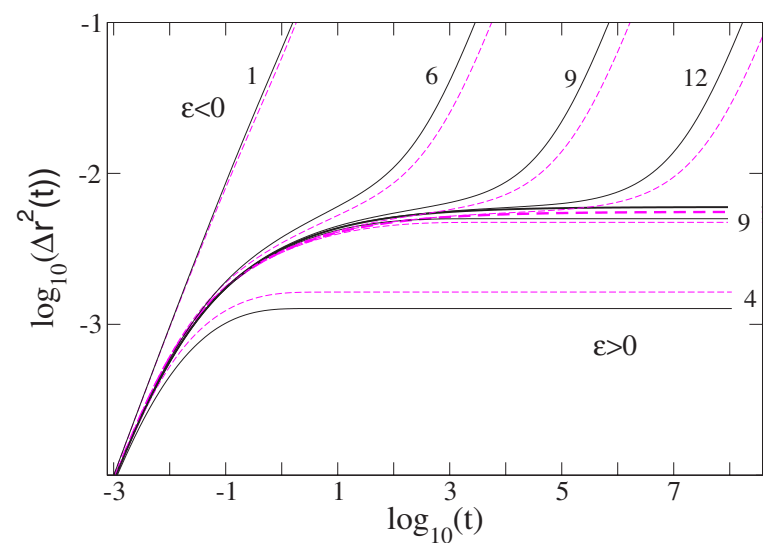

FIG. 14. (Color online) Scaled mean-squared displacement $\Delta r^{2}(t)=\delta r^{2}(t) /(2 d)$ in units of $(2 R)^{2}$ for disks in $d=2$ (black, solid line) and spheres in $d=3$ (magenta, dashed line) for different $\varepsilon$ $= \pm 10^{-n / 3}$ as labeled. The thick lines are the critical curves. correlators-i.e., correlators with a large range of $q$ valuescontribute to the memory kernels [35]. This arises for large wave vectors: then $\beta_{q}=b$ holds, and the Kohlrauschrelaxation time depends on wave vector as $\widetilde{\tau}_{q} \sim q^{-1 / b}$. In the opposite limit, when only one correlator contributes to the memory function, the schematic $\mathcal{F}_{2}$ model is obtained, where the $\alpha$ process is exponential [2]. The latter description obviously best applies to the relaxation of the correlator $\widetilde{\phi}_{q_{1}}(\widetilde{t})$ at the position $q_{1}$ of the primary peak in the structure factor. It corresponds to the motion of particles connected to their average separation, which in MCT is predominantly coupled back to itself. Guided by these two limits, we speculate that the Kohlrausch law provides rather good fits to the $\alpha$ process in $d=2$ because the spread of Kohlrausch exponents between $b=0.61 \leqslant \beta_{q}<1$ is smaller in $d=2$ than it is in $d=3$.

Finally, we have calculated the mean-squared displacement $\Delta r^{2}(t)=\delta r^{2}(t) /(2 d)$, weighted with $1 /(2 d)$. Figure 14 presents the results obtained from the solution of Eq. (24) for $d=2$ and $d=3$ [23]. For the liquid phase the increase of $\varphi$ leads to the formation of a plateau which has its origin in the cage effect, independent of $d=2$ or $d=3$. The dynamical behavior for $t$ small is governed by the short-time diffusion constant $D_{0}^{(s)}$ and that for $t \rightarrow \infty$ by $D(\varphi) \sim\left[t_{\sigma}^{\prime}(\varphi)\right]^{-1} \sim\left(\varphi_{c}-\varphi\right)^{\gamma}$, the long-time diffusivity. At $\varphi_{c}$-i.e., for $\varepsilon=0$ - a transition occurs where the cage has an infinite life time such that $D(\varphi)=0$ for $\varphi \geqslant \varphi_{c}$. Consequently the particle becomes localized with finite localization length $r_{s}$ [cf. Eq. (27)]. It is interesting that $r_{s}^{d=2} \cong 0.077(2 R)$ and $r_{s}^{d=3} \cong 0.075(2 R)$ are almost the same, as can be seen from Fig. 14 (as well as from Fig. 3).

\section{COMPARISON WITH EXPERIMENT}

Although our results were obtained for monodisperse hard disks, we have made a comparison of the mean-squared displacement (MSD) $\delta r^{2}(t)$ with corresponding experimental results of the binary system studied in Ref. [19].

The experimental system consists of a two-dimensional binary mixture of superparamagnetic polystyrene particles doped with $\mathrm{Fe}_{2} \mathrm{O}_{3}$ clusters, confined by gravity to a water-air interface. This is realized by a free-hanging flat water droplet in a top-sealed glass cylinder. Aggregation of the particles is prevented by addition of sodium dodecyl sulfate. The system can be considered truly as two dimensional because the gravitational lengths are small compared to the particle diameters $\left(2 R_{\text {small }}=2.8 \mu \mathrm{m}, 2 R_{\mathrm{big}}=4.7 \mu \mathrm{m}\right)$. An inclination control ensures the horizontal stability by $\pm 1 \mu \mathrm{rad}$ and the flatness of the interface can be controlled in a range less than $1 \mu \mathrm{m}$ relative to $6 \mathrm{~mm}$ sample diameter [36]. We control the interaction by an external magnetic field that induces magnetic moments in each particle. The magnetic dipole interaction dominates the pair potential. The sample is observed by video microscopy with a charge-coupled-device (CCD) camera. Within an area of $1 \mathrm{~mm}^{2}$ the trajectories of $2100 \mathrm{big}$ and 900 small particles are recorded for several days. It is possible to observe the dynamics of the system from fluid to glassy dependent on the applied magnetic field. Local and statistical information can be obtained on all relevant length 


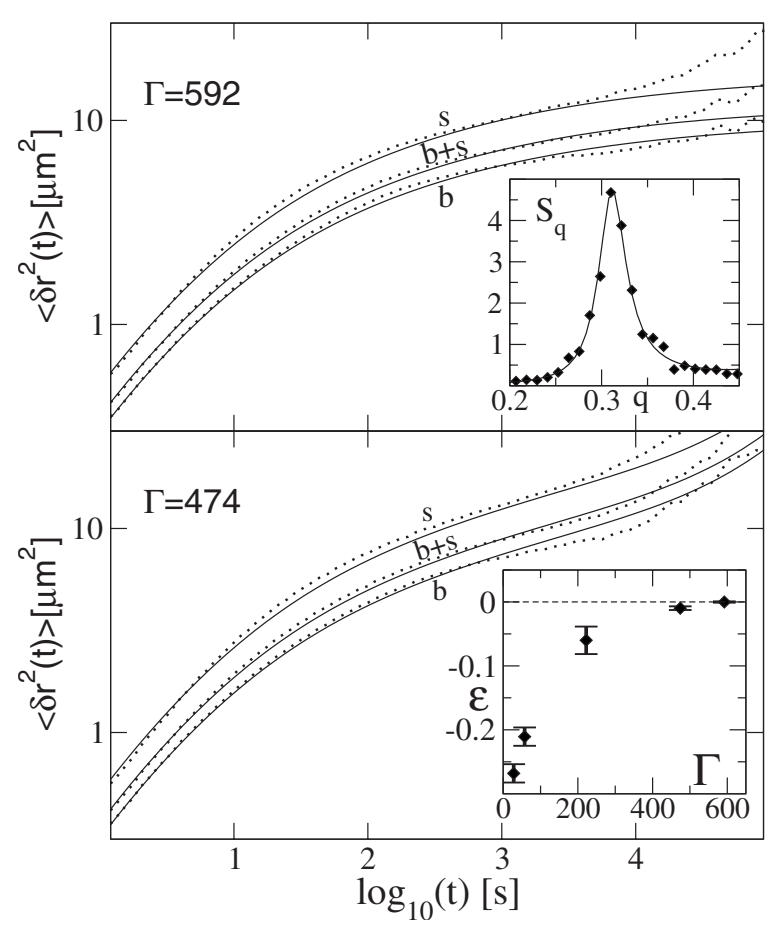

FIG. 15. Comparison of experimental data (dotted line) from [36] with theoretical curves (solid). Since the experimental system is a binary mixture three MSD curves are measured. Curve $b$ is measured considering only big particles, $s$ only small particles and $b+s$ takes all particles into account without discerning big or small. The top panel is at $\Gamma=592, \varepsilon=-10^{-3}$, the bottom one at $\Gamma=474$, $\varepsilon=-10^{-2}$. The data at $\Gamma=592$ can in the given region also be fitted by a critical curve and could thus correspond to a state in the glass. At $\Gamma=592$, the unit of time is fitted as $\tau^{\mathrm{BD}}=1 / 470 \mathrm{~s}$, and the unit of length is fitted as $(420,700,500) \mu \mathrm{m}^{2}$ for the big (curve $b$ ), the small (curve $s$ ), and all particles (curve $b+s$ ). This leads to a short time diffusion coefficient $D_{0} \approx 0.9 e^{-13} \mathrm{~m}^{2} / \mathrm{s}$ and an effective diameter of $2 R_{\mathrm{eff}}^{\mathrm{big}} \approx 20.5 \mu \mathrm{m}$ for the big particles. The lower inset shows the fitted separation parameter $\varepsilon$ versus $\Gamma$. The upper inset shows a comparison of the (partial) structure factor of the big particles at $\Gamma=592$ with the one of the hard disk system at $\varepsilon=0$; fortuitously, deviations only appear at larger $q$.

and time scales. A more detailed description of an earlier variant of this system can be found in [19].

The control parameter varied in the experimental system is the interaction parameter, or inverse temperature, $\Gamma$. Interaction parameters larger than $\Gamma_{m}=60$ are used, the value at which the corresponding monodisperse experimental system forms a crystal. Because we aim for a qualitative test of our MCT results only, like the existence of finite localization length at the transition, we concentrate on the $\varepsilon$-insensitive parts of the MSDs. Thus, we arbitrarily choose $\varepsilon \approx 0$ for the experimental curve at the highest $\Gamma$ available, $\Gamma=592$. The result of this comparison is shown in Fig. 15.

In the fit a global length and time scale was obtained by fitting the $\Gamma=592$ data. The length and time scales found at this $\Gamma$ are then used in fits to data for lower $\Gamma$. Quite good matching to the data is achieved as shown in Fig. 15 for the example of $\Gamma=474$. Hence the scaling factors for time and length scales can be taken as constants and independent of parameter $\Gamma$. With the scales set, the only fitting parameter left is $\varphi$, which is adjusted by eye. We find the fits obtained remarkably good, considering the simplification to map the binary experimental system onto monodisperse hard disks. From the fitted length scale of the single particle MSD of the majority particles (viz., the big ones) a reasonable value for the effective particle size follows. The position of the primary peak in the hard-disk structure factor closely agrees with the position of the experimentally obtained (partial) structure factor of the big particles; see the upper inset of Fig. 15. We thus conclude that MCT correctly captures the ratio of localization length to average particle distance.

\section{SUMMARY AND CONCLUSIONS}

In a first step we have determined the explicit dependence of the MCT functions $\mathcal{F}_{q}\left[\phi_{k}\right]$ and $\mathcal{F}_{q}^{(s)}\left[\phi_{k}, \phi_{k}^{(s)}\right]$ on the spatial dimensionality $d$. This has also been done in the hydrodynamic limit $q \rightarrow 0$, which generalizes the well-known result for $d=3[1,22,23]$ to arbitrary dimensions.

The major motivation of the present contribution has been the investigation of the existence of a dynamic glass transition in two-dimensional systems. As model system we have chosen monodisperse hard disks which might be a reasonable approximation for a system of polydisperse hard disks, at least on a qualitative level. Taking the static input quantity $S_{q}$ from a modified, hypernetted chain approximation we have found that an ergodic-nonergodic transition occurs at a critical packing fraction $\varphi_{c}^{d=2} \cong 0.697$. At this critical density, both the fluctuations of the collective and self-density simultaneously freeze into a glassy state, as for $d=3$. That $\varphi_{c}^{d=2}$ is about $35 \%$ above $\varphi_{c}^{d=3}$ might be not surprising, since the packing fraction $\varphi_{\text {triang }}^{d=2}=\pi / \sqrt{12} \cong 0.9069$ of the triangular lattice is also larger than $\varphi_{\mathrm{hcp}}^{d=3}$, the value for the hexagonal close-packed lattice, by about $20 \%$. The difference between $\varphi_{c}^{d=2}$ and $\varphi_{c}^{d=3}$ becomes even more obvious when scaling is done with the random close packing values $\varphi_{\text {rcp }}^{d=2} \cong 0.84$ and $\varphi_{\text {rcp }}^{d=3} \cong 0.64$ [37]. $\left(\varphi_{c} / \varphi_{\text {rcp }}\right)^{d=2} \cong 0.83$ and $\left(\varphi_{c} / \varphi_{\text {rcp }}\right)^{d=3} \cong 0.81$ deviate by not more than $5 \%$. Consequently, $\varphi_{\text {rcp }}^{d}$ might be a reasonable scale for $\varphi_{c}^{d}$. So far MCT applied to $d=2$ provides an explanation for the dynamic glass transition observed in Refs. [11-13]. Since MCT overestimates the glass transition, it is not a surprise that $\varphi_{c}^{\operatorname{sim}} \approx 0.80$ is above $\varphi_{c}^{d=2} \cong 0.697$, quite similar to $d=3$ [3].

For all the investigated quantities and properties connected to the local "cage motion" on an intermediate time window, like the critical nonergodicity parameter, critical amplitudes, the exponent parameter, the amplitudes $K_{q}$ and $\bar{K}_{q}$ of the next-to-leading order corrections, etc., we have found a weak dependence on dimensionality only, comparing $d=2$ and $d=3$. The largest change between $d=2$ and $d=3$ occurs for the coherent NEP at higher wave vectors. For the two-dimensional case, the $q$ dependence is more abrupt. Otherwise, the similarity also holds for the localization length $r_{s}$ which differs by less than 3\%. Accordingly, the Lindemann criterion applied to the melting of the glass phase is almost $d$ independent, at least for $d=2$ and $d=3$. We consider this an intriguing finding, considering the role of dimensionality in how hydrodynamic back flow affects diffusion [38] and the 
dependence of thermodynamic glass transitions on dimension $[5,6]$. Moreover, the Lindemann criterion does not apply to freezing into ordered states in $d=2$, as has been observed also in the experimental system studied here [39]. For longer times, we found the "(time-temperature-)superposition principle" of the $\alpha$ process; the correlators collapse onto a nonexponential master function. Interestingly, we observed for hard disks that the functional form of the $\alpha$ relaxation closely resembles a Kohlrausch law. This holds better in $d=2$ than for hard spheres in $d=3$. While the prediction of a superposition principle for the final decay is guaranteed by the general structure of the MCT equations, the shape of the relaxation process provides information on the local particle rearrangements. Apparently, in the lower dimension the memory kernels arise from a large number of contributions so that the Kohlrausch law as a limiting law of large numbers provides a better approximation to the cooperative motion during the $\alpha$ decay in $d=2$ than in $d=3$. There is another conclusion we can draw from our results. On a qualitative level, we have found consistency with the observations made in Refs. [15-17]. Particularly, the stretching found for the monodisperse Lennard-Jones system [16] and the binary mixture of soft disks [17] as well as the two-step relaxation process [17] can be described by MCT in two dimensions.

Of course, more quantitative comparisons are necessary. MCT should also be worked out for binary hard disks without and with pair interactions. This will allow to compare MCT results with the experimental ones [19] in detail. A first attempt has been done concerning the mean-squared displacement. A more or less satisfactory agreement has been found over about four decades in time (see Fig. 15). Whether the systematic discrepancies between the theoretical and the experimental result can be attributed to the different two model systems-i.e., on one side the monodisperse hard disks and on the other the binary hard disks with dipolar repulsion at rather low densities-is one of the open questions we intend to study in the future.

\section{ACKNOWLEDGMENTS}

We thank U. Gasser, P. Keim, W. van Megen, and Th. Voigtmann for helpful discussions and acknowledge support by the Deutsche Forschungsgemeinschaft in SFB 513 and IRTG 667, Sp714/3, and NSF Grants Nos. DMT0137119 and DMS0244492.
[1] U. Bengtzelius, W. Götze, and A. Sjölander, J. Phys. C 17, 5915 (1984).

[2] W. Götze, in Liquids, Freezing and the Glass Transition, edited by J. P. Hansen, D. Levesque, and J. Zinn-Justin (NorthHolland, Amsterdam, 1991).

[3] W. Götze and L. Sjögren, Rep. Prog. Phys. 55, 241 (1992).

[4] W. Kob, J. Phys.: Condens. Matter 11, R85 (1999); arXiv:cond-mat/0212344; W. Götze, J. Phys.: Condens. Matter 11, A1 (1999).

[5] M. Mézard and G. Parisi, J. Phys. A 29, 6515 (1996).

[6] M. A. Moore and J. Yeo, Phys. Rev. Lett. 96, 095701 (2006).

[7] R. Schilling, in Collective Dynamics of Nonlinear and Disordered Sytems, edited by G. Radons, W. Just, and P. Häussler (Springer, Berlin, 2005).

[8] H. J. Schöpe, G. Bryant, and W. van Megen, Phys. Rev. Lett. 96, 175701 (2006).

[9] M. D. Rintoul and S. Torquato, Phys. Rev. Lett. 77, 4198 (1996).

[10] S. R. Williams, I. K. Snook, and W. van Megen, Phys. Rev. E 64, 021506 (2001).

[11] L. Santen and W. Krauth, Nature (London) 405, 550 (2000).

[12] L. Santen and W. Krauth, e-print arXiv:cond-mat/0107459.

[13] B. Doliwa and A. Heuer, Phys. Rev. E 61, 6898 (2000).

[14] A. Donev, F. H. Stillinger, and S. Torquato, Phys. Rev. Lett. 96, 225502 (2006).

[15] F. Lançon and P. Chaudhari, in Computer-Based Microscopic Description of the Structure and Properties of Materials, edited by J. Broughton, W. Krakow, and S. T. Pantelides, Mater. Res. Soc. Symp. Proc. (Materials Research Society, Pittsburgh, 1986), p. 95.

[16] S. Ranganathan, J. Phys.: Condens. Matter 6, 1299 (1994).

[17] D. N. Perera and P. Harrowell, Phys. Rev. E 59, 5721 (1999).

[18] W. Kob and H. C. Andersen, Phys. Rev. E 51, 4626 (1995); ibid. 52, 4134 (1995).
[19] H. König, R. Hund, K. Zahn, and G. Maret, Eur. Phys. J. E 18, 287 (2005).

[20] W. Götze, E. Leutheusser, and S. Yip, Phys. Rev. A 23, 2634 (1981); Phys. Rev. A 25, 533 (1982).

[21] E. V. Zenkov, Europhys. Lett. 69, 390 (2005).

[22] T. Franosch, M. Fuchs, W. Götze, M. R. Mayr, and A. P. Singh, Phys. Rev. E 55, 7153 (1997).

[23] M. Fuchs, W. Götze, and M. R. Mayr, Phys. Rev. E 58, 3384 (1998).

[24] J.-L. Barrat, W. Götze, and A. Latz, J. Phys.: Condens. Matter 1, 7163 (1989).

[25] J. P. Hansen and I. R. McDonald, Theory of Simple Liquids (Academic Press, London, 1986).

[26] M. P. Allen and D. J. Tildesley, Computer Simulation of Liquids (Oxford University Press, Oxford, 1987).

[27] F. Lado, J. Chem. Phys. 49, 3092 (1968).

[28] M. Baus and J-L. Colot, J. Phys. C 19, L643 (1986).

[29] Y. Rosenfeld, Phys. Rev. A 42, 5978 (1990).

[30] Y. Rosenfeld and N. Ashcroft, Phys. Lett. 73A, 31 (1979).

[31] T. M. Truskett, S. Torguato, S. Sastry, P. G. Debenedett, and F.H. Stillinger, Phys. Rev. E 58, 3083 (1998).

[32] M. Fuchs, W. Götze, I. Hofacker, and A. Latz, J. Phys.: Condens. Matter 3, 5047 (1991)

[33] K. Kawasaki, Transp. Theory Stat. Phys. 24, 755 (1995).

[34] M. Fuchs, I. Hofacker, and A. Latz, Phys. Rev. A 45, 898 (1992).

[35] M. Fuchs, J. Non-Cryst. Solids 172-174, 241 (1994).

[36] F. Ebert and G. Maret (unpublished).

[37] J. G. Berryman, Phys. Rev. A 27, 1053 (1983); T. S. Majmudar, M. Sperl, S. Luding, and R. P. Behringer, Phys. Rev. Lett. 98, 058001 (2007).

[38] M. A. van der Hoef and D. Frenkel, Phys. Rev. Lett. 66, 1591 (1991).

[39] K. Zahn and G. Maret, Phys. Rev. Lett. 85, 3656 (2000). 